\title{
RETRIEVAL OF MINERAL ABUNDANCES OF THE DELTA REGION IN EBERSWALDE, MARS
}

\author{
Xing Wu ${ }^{\text {a,b }}$, Xia Zhang ${ }^{a^{*},}$ Honglei Lin ${ }^{a, b}$ \\ a. Institute of Remote Sensing and Digital Earth, Chinese Academy of Sciences, Beijing, 100101, China \\ (wuxing, zhangxia, linhonglei)@ radi.ac.cn \\ ${ }^{\mathrm{b}}$.University of Chinese Academy of Science, Beijing,100049, China
}

Commission III, ICWG III/II

KEY WORDS: Mars, Eberswalde Crater, Minerals, Collaborative sparse unmixing, Abundance retrieval

\begin{abstract}
:
Eberswalde Crater, a hotspot of Mars exploration, possesses an unambiguous hydrological system. However, little research has been performed on the large-scale mineral abundances retrieval in this region. Hence, we employed hyperspectral unmixing technology to quantitatively retrieve mineral abundances of the delta region in Eberswalde. In this paper, the single-scattering albedos were calculated by the Hapke bidirectional reflectance function from Compact Reconnaissance Imaging Spectrometer for Mars (CRISM) data (FRT000060DD) and CRISM spectral library respectively, and a sparse unmixing algorithm was adopted to quantitatively retrieve mineral abundances. The abundance maps show that there are six kinds of minerals (pyroxene, olivine, plagioclase, siderite, diaspore, and tremolite). By comparing minerals spectra obtained from images with corresponding spectra in spectral library, we found the similar trend in both curves. Besides, the mineral abundance maps derived in this study agree well spatially with CRISM parameter maps. From the perspective of mineralogy, the instability of pyroxene and olivine indicates the area in which they distribute is close to provenance, and the original provenance is ultrabasic rock(e.g. peridotite) and basic rock(e.g. gabbro), respectively. And minerals, existing in the area of alluvial fan, also distribute in the outside of alluvial fan, which might be caused by fluid transportation.
\end{abstract}

\section{INTRODUCTION}

The fan delta in Eberswalde crater has been interpreted as strong evidence for sustained liquid water on early Mars(Malin and Edgett 2003; Mangold et al., 2012; Pondrelli et al., 2014). Previous study mainly focus on the morphology, stratigraphy, and geology evolution of this place(Lewis \& Oded 2006; Mangold et al., 2012; Pondrelli et al., 2008). Although these research provide clues of their geology history, mineral identification and abundance retrieval can further provide unique constraints on their formation environment(Liu et al., 2016). Poulet et al (2014) modeled the mineral abundances by average spectrum of Compact Reconnaissance Imaging Spectrometer for Mars (CRISM) 121 pixels over the Eberswalde deltaic deposits. However, little research has been performed on the large-scale minerals retrieval in this region. Spectral unmixing aims at extracting the spectrally pure constituent materials and their respective fractional abundances in each pixel of a hyperspectral image scene(Bioucas-Dias et al., 2012). Hence, we employed a spectral unmixing technology to quantitatively retrieve mineral abundances of the delta region in Eberswalde.

There are several efforts have been made to attempt quantitative mineral abundances based on visible near infrared (VNIR) imaging spectroscopy on Mars. In the VNIR, the observed signal of reflected light from a particulate surface is a non-linear combination of the spectral properties of the minerals (Goudge et al., 2015).The widely used non-linear mixing models in planetary spectra study are Hapke radiative transfer model(Hapke 1981) and the geometric optics model of Shkuratov (Shkuratov et al., 1999). Both the Hapke and Shkuratov models have been used to retrieve mineral abundances in VNIR spectra of Mars(Liu et al., 2016; Poulet et al., 2014).

In this study, we used Hapke radiative transfer model to convert CRISM I/F to single-scattering albedos, so as to perform linear unmixing to Martain minerals spectra. It's challenging to find pure spectral signatures(endmembers) in practice(Iordache et al., 2011). Iordache et al (2011) proposed a sparse unmixing algorithm which sidesteps endmember extraction process, it aims at finding the optimal subset of signatures in a spectral library that can best model each mixed pixel in the scene. Therefore, we adopted a sparse unmixing algorithm to quantitatively retrieve mineral abundances by using image obtained by CRISM together with CRISM spectral library.

\section{STUDY AREA AND DATA SETS}

\subsection{Study Area}

Eberswalde Crater centered at $24^{\circ} \mathrm{S}, 33^{\circ} \mathrm{W}$, is a partially buried impact crater located north-northeast of the Holden crater. Its diameter is about $65.3 \mathrm{~km}$. Geomorphology provide unambiguous evidence that water was present and stable here during some part of its history (Pondrelli et al., 2014). It possesses a fan delta, fluvial channels. According to stratigraphy of cross cutting relations, Pondrelli et al (2008) interpreted there are five different lobes, and each lobe corresponds to a different stage of fan delta evolution. It was one of the final four proposed landing sites for the Mars rover Mars Science Laboratory (MSL) mission. Study on minerals and their abundances can further enhance our knowledge on their deposition process. 

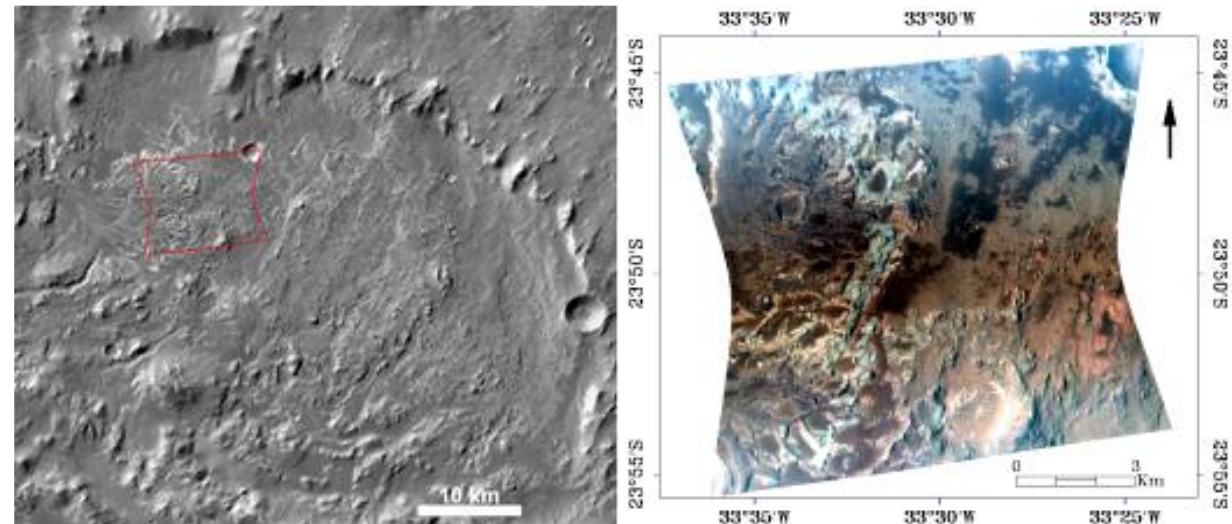

Figure 1. Eberswalde Crater.(a) Overview of Eberswalde Crater, which is a partially buried impact crater. Image is a mosaic of CTX images, overlain on the THEMIS $100 \mathrm{~m} /$ pixel global daytime infrared mosaic. CRISM FRT000060DD footprint is shown outlined in red. (b) CRISM image FRT000060DD (R:2.529 $\mu \mathrm{m}, \mathrm{G}: 1.506 \mu \mathrm{m}, \mathrm{B}: 1.080 \mu \mathrm{m})$. North is up in both images.

\subsection{Data Sets}

This paper intent to study minerals of delta region. Therefore we emphasize CRISM hyperspectral data and CRISM spectral library, which help to distinguish minerals and retrieve their abundances.

\subsubsection{CRISM data}

CRISM is a VNIR imaging spectrometer onboard the Mars Reconnaissance Orbiter (MRO) that covers the wavelength range of $0.36 \sim 3.94 \mu \mathrm{m}$. In this paper, we used one targeted mode observation, which has a full spatial resolution (FRT) of 18 $\mathrm{m} / \mathrm{pixel}$, and a spectral sampling of $6.5 \mathrm{~nm} / \mathrm{channel(Murchie} \mathrm{et}$ al., 2007).

In this work, we downloaded the CRISM I/F data from Planetary Data System (PDS). CRISM data processed to I/F through the ratio of the measured sensor radiance and a solar irradiance divided by $\pi$ (McGuire et al., 2009a). Images were photometrically corrected by dividing each spectrum by the cosine of the incidence angle. Then they were atmospherically corrected using the "Volcano Scan" method(McGuire et al., 2009a). Although Volcano Scan technique does not remove aerosol contributions to spectra completely, it corrects the main atmospheric absorption caused by $\mathrm{CO}_{2}$ (Mcguire et al., 2009b). We applied filter to remove both spatial and spectra noise in images. Finally, we made geometric correction by projection. All above operations were performed by CRISM Analysis Toolkit. Because minerals primary spectral feature are within $1 \sim 2.6 \mu \mathrm{m}$, and avoid surface thermal emission at wavelengths $>2.6 \mu \mathrm{m}$, hence, the bands befor $1 \mu \mathrm{m}$ and after $2.6 \mu \mathrm{m}$ were not used.

\subsubsection{CRISM Spectral Library}

The CRISM spectral library is a collection of laboratory spectra of Mars-analog materials supplied by the CRISM Team. The library was used for pure endmembers in spectral unmixing. It contains 2463 spectral analyses of 1228 specimens, all measured under desiccating conditions to remove atmospheric $\mathrm{H} 2 \mathrm{O}$ contamination and provide a better proxy to current desiccated Martian conditions(Lin et al., 2015). We chose the naturally occurring minerals and retained valid minerals spectra, 768 samples in total. All spectra were resampled at the wavelength of the CRISM using linear interpolation.

\section{METHODS}

\subsection{Single-Scattering Albedo Retrieval}

Single-Scattering Albedo(SSA) is the ratio of scattered light to total extincted light. Hapke bidirectional reflectance distribution function relates the reflectance of a mixture to a linear combination of the SSA of its constituent endmembers(Hapke, 1981).It enables us to perform linear mixture analysis of the observed spectra(Goudge et al., 2015; Lin and Zhang 2017; Liu et al., 2016). The function is described as the following equation(Hapke, 1993):

$$
\begin{gathered}
r(i, e, g)=\frac{\omega}{4} \frac{\mu_{0}}{\left(\mu_{0}+\mu\right)}\left[(1+B(g)) P(g)+H\left(\mu_{0}\right) H(\mu)-1\right] \\
H(\mu)=\frac{1+2 \mu}{1+2 \mu \sqrt{1-\omega}}
\end{gathered}
$$

where $r(i, e, g)$ is the radiance factor, equivalent to CRISM I/F; $i, e$, and $g$ are incidence, emergence, and phase angles, respectively; $w$ is the average single particle scattering albedo; $B(g)$ is the backscattering function at the phase angle $\mathrm{g} ; P(g)$ is the surface phase function; $\mathrm{H}$ is the Chandrasekhar integral function associated with the observation geometry.

In this work, we referred to (Goudge et al., 2015), assumed that there is no opposition effect, $B(g)=0$; and also assumed that $P(g)=1$, which implies that the surface scatters isotropically. Because the opposition effect is strongest for phase angles $<15^{\circ}$ (Mustard \& Pieters, 1989), and our data are at phase angles $\geq 30^{\circ}$. The effect of anisotropic scattering on abundance estimates for binary and ternary mixtures is about $5-10 \%$ when measured at phase angles of $15-120^{\circ}$ (Mustard \& Pieters, 1989). We can calculate analytic solution for SSA based on equation (1), (2) and $B(g)=0, P(g)=1$. Therefor, we converted reflectance of CRISM images and spectral library to SSA, respectively.

\subsection{Sparse Unmixing Algorithm}

Sparse unmixing is an active research area in hyperspectral unmixing in recent years(Giampouras et al., 2015; Iordache et al., 2011, 2012, 2014a; Iordache et al., 2014b; Shi et al., 2014; Tang et al., 2015), which aims to find the optimal subset of signatures in a spectral library that can best model hyperspectral data. A sparsity regularizer is commonly imposed to promote the number 
of selected signatures as small as possible. This sparse unmixing problem can be writen as equation(3)(Iordache et al., 2014a):

$$
\min _{x}\|A X-Y\|_{F}+\lambda\|X\|_{2,1}+\kappa_{R+}(X)
$$

where $Y$ is the measured spectra of the pixels ( $L$ bands, $N$ pixels), $Y \in R^{L \times N} ; X$ is the abundance matrix corresponding to reflectance spectral library $A$ ( $L$ bands, $M$ signatures), $A \in R^{L \times M}$, $X \in R^{M \times N} ;\|X\|_{2,1}=\sum_{k=1}^{M}\left\|x^{k}\right\|_{2}$ to denote the $\ell_{2,1}$ norm, this term imposes sparsity among the endmembers simultaneously for all pixels; $\kappa_{R+}(X)=\sum_{j=1}^{n} \kappa_{R_{+}}(x), \quad x_{i}$ represents the $i$-th column, this term is a indicator function $\left(\kappa_{R+}\left(x_{i}\right)\right.$ is zero if $x_{i}$ belongs to the nonnegative orthant, otherwise $\kappa_{R+}\left(x_{i}\right)$ is infinity).

We adopted this algorithm, the main steps in this work are the following ones : (1) To infer the subspace in which the CRISM data lives by using the hyperspectral signal subspace identification by minimum error (HySime) algorithm(BioucasDias \& Nascimento, 2008).(2) All the mineral spectra of the spectral library were projected onto the subspace and the projection errors for each mineral spectra were calculated.(3) The spectra with smaller projection error were then retained as the endmember which truly contributed to the observed mixtures. (4) A sparse unmixing algorithm (Iordache et al., 2014a) was applied to retrieve the minerals abundance of the delta region in Eberswalde.

\section{RESULTS}

\subsection{Spectral Unmixing Results}

The abundance maps show that there are five kinds of primary minerals (pyroxene, olivine, plagioclase, siderite, diaspore) and one kind of alteration minerals (tremolite). As shown in Figure 2, pyroxene is mainly distributed in the front of alluvial fan. Olivine is distributed in the central area, shown as east-west distribution. Plagioclase is mainly distributed in the western delta region and a small crater edge in the northeastern corner. Siderite, with relatively less content, is mainly distributed along the edge of alluvial fan. Diaspore is mainly distributed along the river valley and the relatively enriched diaspore in the northeastern corner was probably caused by transportation of liquid water in the past. Tremolite is distributed in the northeastern corner of the delta region.

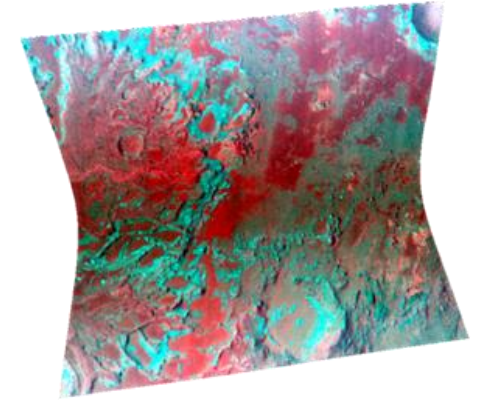

(a) Plagioclase

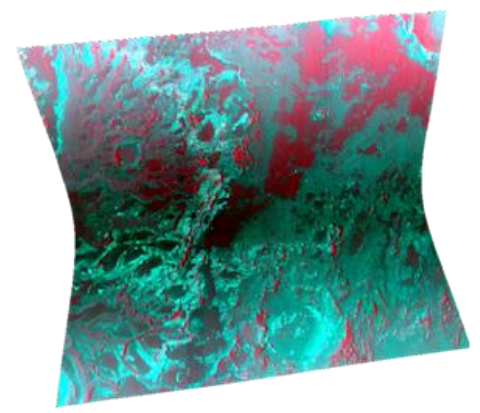

(d) Diaspore

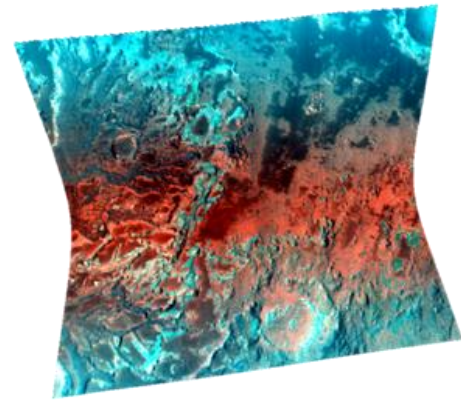

(b) Olivine

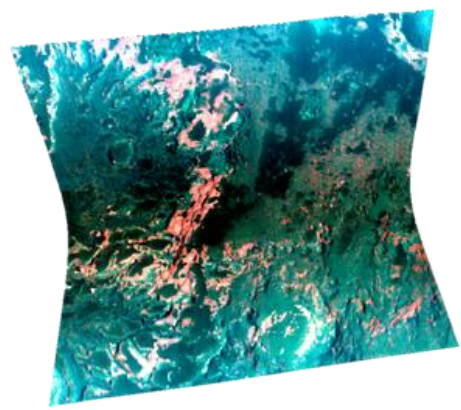

(e) Pyroxene

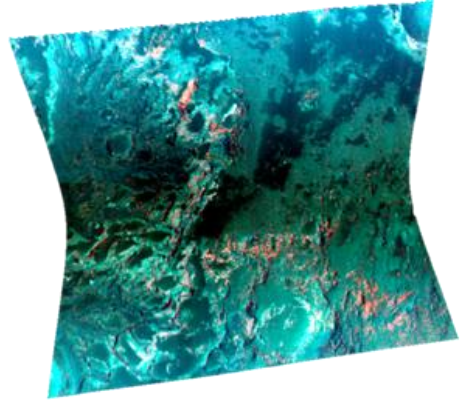

(c) Siderite

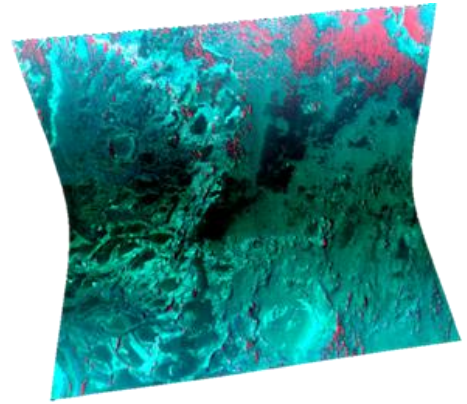

(f) Tremolite

Figure 2. Abundance maps of minerals(R: Abundance map, G: $1.506 \mu \mathrm{m}, \mathrm{B}: 1.080 \mu \mathrm{m})$. The minerals are in red areas.

\subsection{Validation of Results}

We used the ratioing technique to remove residual atmospheric or instrument-related spectral features. Spectra from high abundance areas were extracted and ratioed against areas with spectrally bland material at the same column. By comparing ratioed spectra with corresponding spectra in CRISM library, we found the similar trend in both curves. The features of diaspore(Figure 3c) are not obvious. It's likely that the presence of dust partly masks mineral features.
In order to validate the spatial distribution of minerals, scatterplots of CRISM index values(BD1300, LCPINDEX, OLINDEX)(Viviano-Beck et al., 2014) versus derived major mineral abundance were produced to evaluate the correlation between them. Comparisons of spectral index map and abundance map are shown in Figure 4. The mineral abundance maps derived in this study agree well spatially with CRISM parameter maps. Particularly, the pyroxene abundance map nicely matches the index map of LCPINDEX, and the scatterplot also shows strong correlation. There are minor discrepancies between olivine abundance map and OLINDEX map in Figure 4 
(red circle areas), a possible explanation is that olivine index may overestimates the distribution of olivine when pyroxene is present(Viviano-Beck et al., 2014).
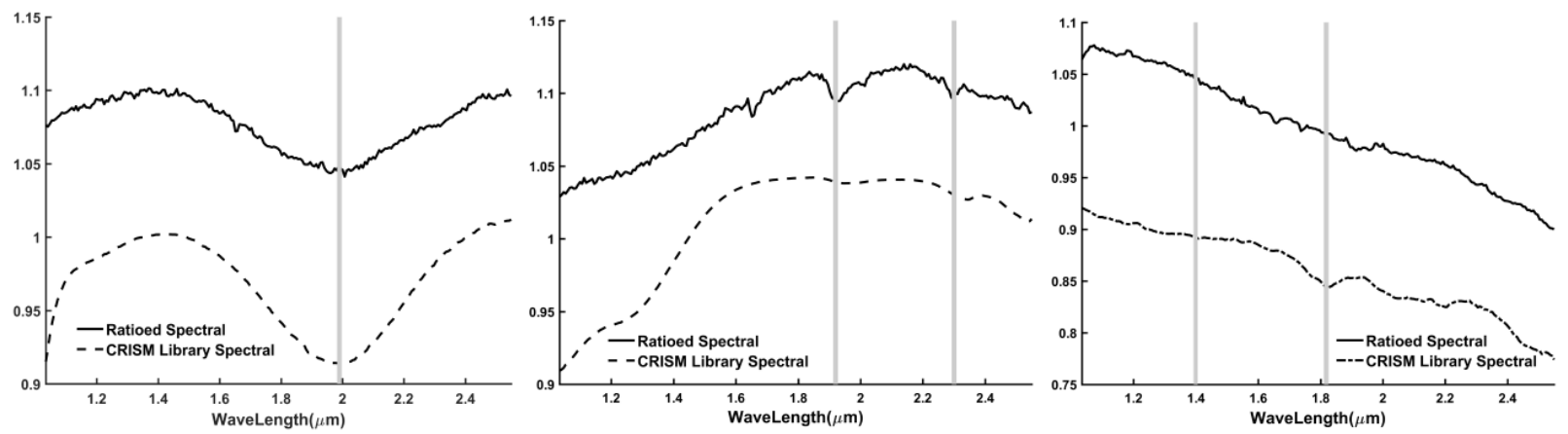

Figure 3. Ratioed spectra and CRISM library spectral from pyroxene(a), siderite(b) and diaspore(c) respective. The grey vertical lines indicate different absorpation feature.

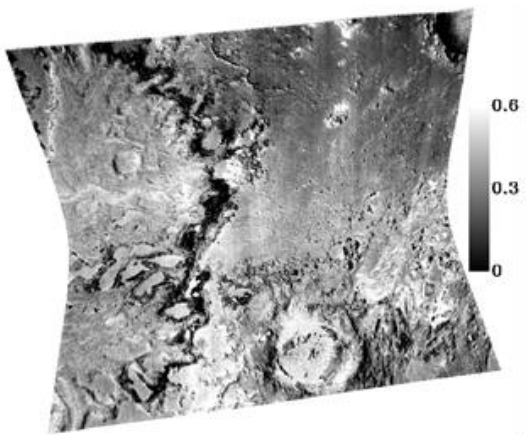

(a) Abundance map of plagioclase

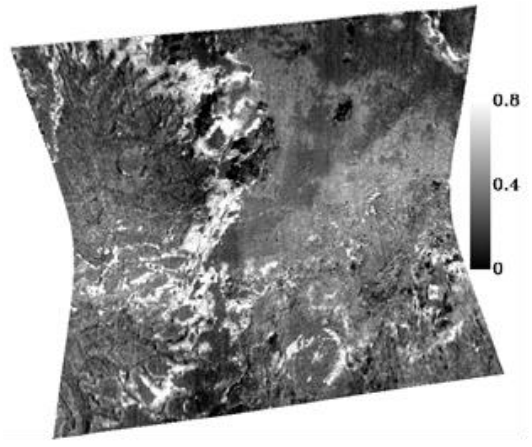

(d) Abundance map of pyroxene

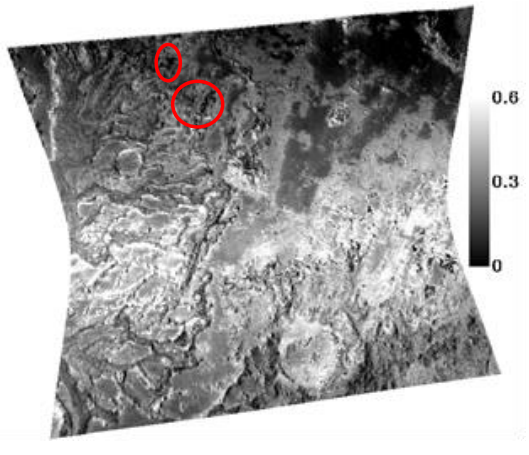

( g) Abundance map of olivine

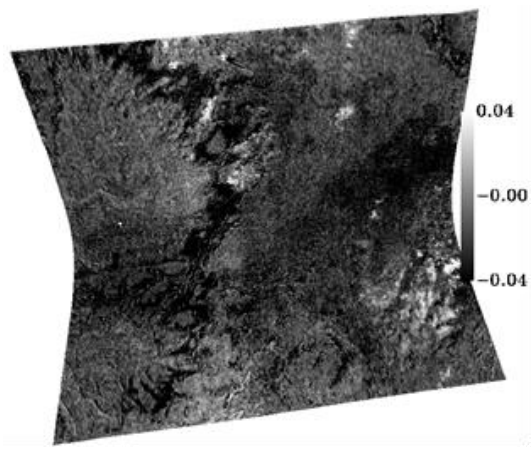

(b) Index map of plagioclase

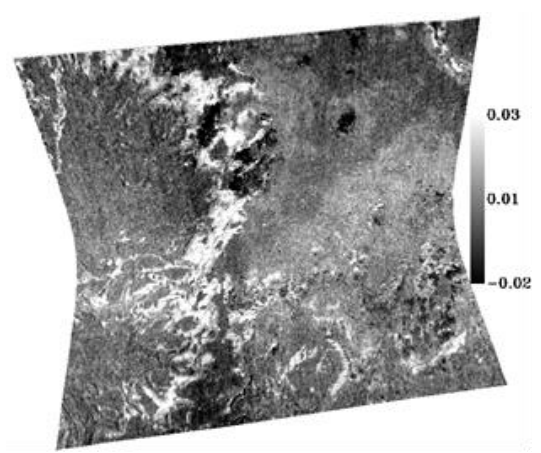

(e) Index map of pyroxene

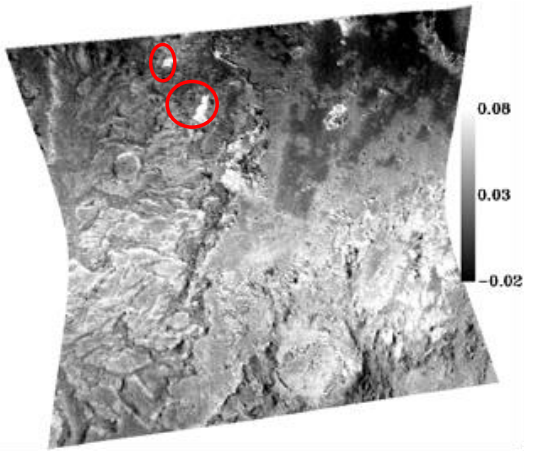

(h) Index map of olivine

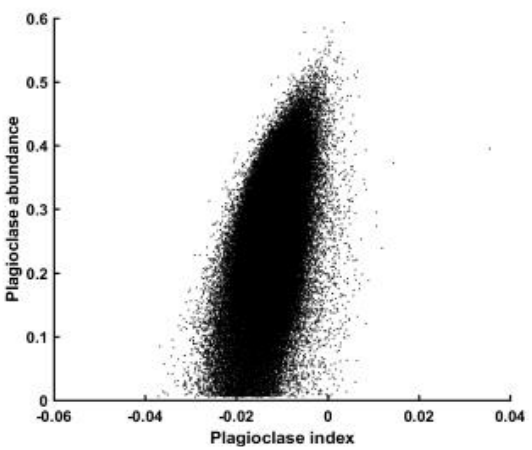

(c) Scatterplot of plagioclase

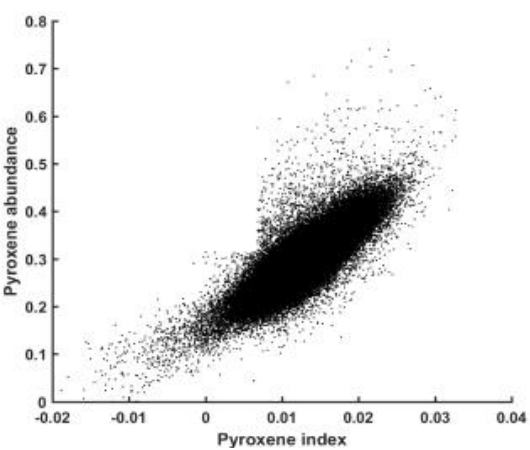

(f) Scatterplot of pyroxene

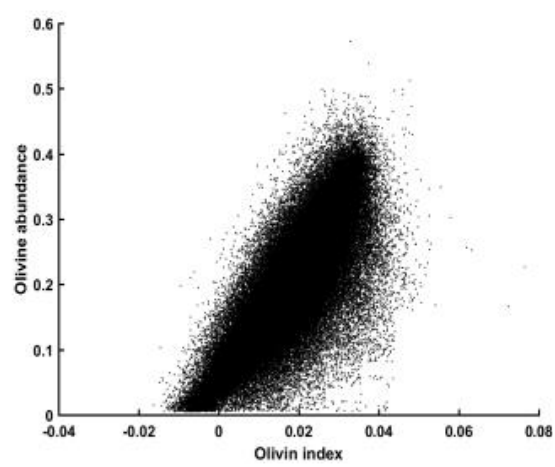

(i) Scatterplot of olivine 
Figure 4. Comparison of spectral index map and abundance map. The red circle ares in $(\mathrm{g})$, (h) are minor discrepancies between olivine abundance map and OLINDEX map.

\section{CONCLUSION}

In this paper, we first used Hapke model to convert CRISM I/F to single-scattering albedos(SSA), then adopted a sparse unmixing algorithm to quantitatively retrieve mineral abundances of the delta region in Eberswalde. Six kinds of minerals have been found, including pyroxene, olivine, plagioclase, siderite, diaspore and tremolite. From the perspective of mineralogy, the instability of pyroxene and olivine indicate the area in which they distribute are close to provenance, and the original provenance are ultrabasic rock(e.g. peridotite) and basic rock(e.g. gabbro), respectively. Tremolite may be formed in contact metamorphism, and the magma provides high temperature containing $\mathrm{Si}$ fluid, carbonate surrounding rock provides $\mathrm{Ca}$. Although the unmixing results are encouraging, we have to take such problems into consideration: (1) uncertainty from end-members selection, (2) uncertainty from singlescattering albedo inversion model.

\section{REFERENCES}

Bioucas-Dias, J.M., \& Nascimento, J.M.P. (2008). Hyperspectral Subspace Identification. Geoscience \& Remote Sensing IEEE Transactions on, 46, 2435-2445.

Bioucas-Dias, J.M., Plaza, A., Dobigeon, N., Parente, M., Du, Q., Gader, P., \& Chanussot, J. (2012). Hyperspectral Unmixing Overview: Geometrical, Statistical, and Sparse RegressionBased Approaches. IEEE Journal of Selected Topics in Applied Earth Observations \& Remote Sensing, 5, 354-379.

Giampouras, P.V., Themelis, K.E., Rontogiannis, A.A., \& Koutroumbas, K.D. (2015). Simultaneously Sparse and LowRank Abundance Matrix Estimation for Hyperspectral Image Unmixing. IEEE Transactions on Geoscience \& Remote Sensing, $54,4775-4789$.

Goudge, T.A., Mustard, J.F., Head, J.W., Salvatore, M.R., \& Wiseman, S.M. (2015). Integrating CRISM and TES hyperspectral data to characterize a halloysite-bearing deposit in Kashira crater, Mars. Icarus, 250, 165-187.

Hapke, B. (1981). Bidirectional reflectance spectroscopy: 1. Theory. Journal of Geophysical Research Solid Earth, 86, 30393054 .

Hapke, B. (1993). Theory of reflectance and emittance spectroscopy. Cambridge University Press.

Iordache, M.D., Bioucas-Dias, J.M., \& Plaza, A. (2011). Sparse Unmixing of Hyperspectral Data. IEEE Transactions on Geoscience \& Remote Sensing, 49, 2014-2039.

Iordache, M.D., Bioucas-Dias, J.M., \& Plaza, A. (2012). Total Variation Spatial Regularization for Sparse Hyperspectral Unmixing. IEEE Transactions on Geoscience \& Remote Sensing, $50,4484-4502$.

Iordache, M.D., Bioucas-Dias, J.M., \& Plaza, A. (2014a). Collaborative Sparse Regression for Hyperspectral Unmixing. IEEE Transactions on Geoscience \& Remote Sensing, 1, 341354.
Iordache, M.D., Bioucas-Dias, J.M., Plaza, A., \& Somers, B. (2014b). MUSIC-CSR: Hyperspectral Unmixing via Multiple Signal Classification and Collaborative Sparse Regression. IEEE Transactions on Geoscience \& Remote Sensing, 52, 4364-4382.

Lewis, K.W., \& Oded, A. (2006). Stratigraphic analysis of the distributary fan in Eberswalde crater using stereo imagery. Journal of Geophysical Research Planets, 111, 107-109.

Lin, H., \& Zhang, X. (2017). Retrieving the hydrous minerals on Mars by sparse unmixing and the Hapke model using MRO/CRISM data. Icarus, 288, 160-171.

Lin, H., Zhang, X., Shuai, T., Zhang, L., \& Sun, Y. (2015). Abundance retrieval of hydrous minerals around the Mars Science Laboratory landing site in Gale crater, Mars. Planetary \& Space Science, 121, 76-82.

Liu, Y., Glotch, T.D., Scudder, N.A., Kraner, M.L., Condus, T., Arvidson, R.E., Guinness, E.A., Wolff, M.J., \& Smith, M.D. (2016). End - member Identification and Spectral Mixture Analysis of CRISM Hyperspectral Data: A Case Study on Southwest Melas Chasma, Mars. Journal of Geophysical Research Planets.

Malin, M.C., \& Edgett, K.S. (2003). Evidence for persistent flow and aqueous sedimentation on early Mars. Science, 302, 19311934.

Mangold, N., Kite, E.S., Kleinhans, M.G., Newsom, H., Ansan, V., Hauber, E., Kraal, E., Quantin, C., \& Tanaka, K. (2012). The origin and timing of fluvial activity at Eberswalde crater, Mars. Icarus, 220, 530-551.

McGuire, P.C., Bishop, J.L., Brown, A.J., Fraeman, A.A., Marzo, G.A., Frank Morgan, M., Murchie, S.L., Mustard, J.F., Parente, M., Pelkey, S.M., Roush, T.L., Seelos, F.P., Smith, M.D., Wendt, L., \& Wolff, M.J. (2009a). An improvement to the volcano-scan algorithm for atmospheric correction of CRISM and OMEGA spectral data. Planetary and Space Science, 57, 809-815.

Mcguire, P.C., Wolff, M.J., Smith, M.D., Arvidson, R.E., Murchie, S.L., Clancy, R.T., Roush, T.L., Cull, S.C., Lichtenberg, K.A., \& Wiseman, S.M. (2009b). MRO/CRISM Retrieval of Surface Lambert Albedos for Multispectral Mapping of Mars With DISORT-Based Radiative Transfer Modeling: Phase 1-Using Historical Climatology for Temperatures, Aerosol Optical Depths, and Atmospheric Pressures. IEEE Transactions on Geoscience \& Remote Sensing, 46, 4020-4040.

Murchie, S., Arvidson, R., Bedini, P., Beisser, K., Bibring, J.P., Bishop, J., Boldt, J., Cavender, P., Choo, T., \& Clancy, R.T. (2007). Compact Reconnaissance Imaging Spectrometer for Mars (CRISM) on Mars Reconnaissance Orbiter (MRO). Journal of Geophysical Research Atmospheres, 112, 431-433.

Mustard, J.F., \& Pieters, C.M. (1989). Photometric phase functions of common geologic minerals and applications to quantitative analysis of mineral mixture reflectance spectra. Journal of Geophysical Research Solid Earth, 94, 13619-13634.

Pondrelli, M., Rossi, A.P., Marinangeli, L., Hauber, E., Gwinner, K., Baliva, A., \& Lorenzo, S.D. (2008). Evolution and 
depositional environments of the Eberswalde fan delta, Mars. Icarus, 197, 429-451.

Pondrelli, M., Rossi, A.P., Pompilio, L., \& Marinangeli, L. (2014). Application of Sequence-Stratigraphic Concepts to Mars: Eberswalde Crater. Springer International Publishing.

Poulet, F., Carter, J., Bishop, J.L., Loizeau, D., \& Murchie, S.M. (2014). Mineral abundances at the final four curiosity study sites and implications for their formation. Icarus, 231, 65-76.

Shi, Z., Tang, W., Duren, Z., \& Jiang, Z. (2014). Subspace Matching Pursuit for Sparse Unmixing of Hyperspectral Data.
IEEE Transactions on Geoscience \& Remote Sensing, 52, 32563274.

Shkuratov, Y., Starukhina, L., Hoffmann, H., \& Arnold, G. (1999). A Model of Spectral Albedo of Particulate Surfaces: Implications for Optical Properties of the Moon. Icarus, 137, 235-246.

Tang, W., Shi, Z., Wu, Y., \& Zhang, C. (2015). Sparse Unmixing of Hyperspectral Data Using Spectral A Priori Information. IEEE Transactions on Geoscience \& Remote Sensing, 53, 770-783. 